ON INVERSE DYNAMICAL AND SPECTRAL PROBLEMS FOR THE WAVE AND SCHRÖDINGER EQUATIONS ON FINITE TREES. THE LEAF PEELING METHOD.

By

Sergei Avdonin, Victor Mikhaylov, and Karlygash Nurtazina

IMA Preprint Series \#2460

(November 2015)

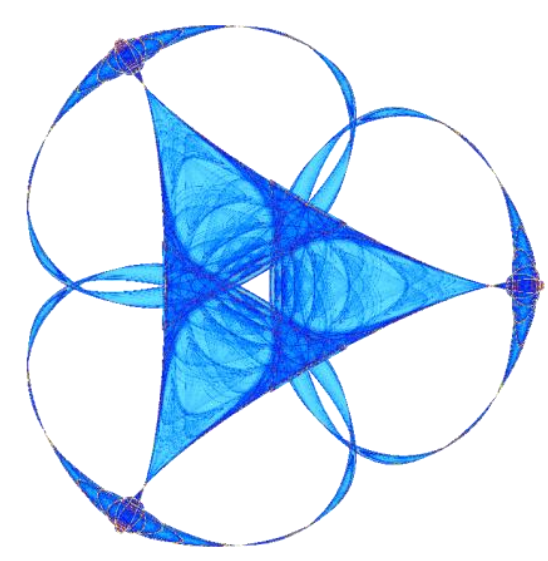

INSTITUTE FOR MATHEMATICS AND ITS APPLICATIONS

UNIVERSITY OF MINNESOTA 400 Lind Hall

207 Church Street S.E.

Minneapolis, Minnesota 55455-0436

Phone: 612-624-6066 Fax: 612-626-7370

URL: http://www.ima.umn.edu 


\title{
On inverse dynamical and spectral problems for the wave and Schrödinger equations on finite trees. The leaf peeling method.
}

\author{
Sergei Avdonin, Victor Mikhaylov, and Karlygash Nurtazina
}

\begin{abstract}
Interest in inverse dynamical, spectral and scattering problems for differential equations on graphs is motivated by possible applications to nano-electronics and quantum waveguides and by a variety of other classical and quantum applications. Recently a new effective leaf peeling method has been proposed by S. Avdonin and P. Kurasov [3] for solving inverse problems on trees (graphs without cycles). It allows recalculating efficiently the inverse data from the original tree to the smaller trees, 'removing' leaves step by step up to the rooted edge. In this paper we describe the main step of the spectral and dynamical versions of the peeling algorithm - recalculating the inverse data for the 'peeled tree'.
\end{abstract}

\section{Introduction.}

Metric graphs with defined on them differential operators or differential equations are called quantum graphs (or differential equation networks). There are two groups of uniqueness results concerning boundary inverse problems for quantum trees. Brown and Weikard [10], Yurko [12], and Freiling and Yurko [11] proved uniqueness results for trees with a priori known topology (connectivity) and lengths of edges using the Titchmarsh-Weyl matrix function (TW-function) as the inverse data.

The second group concerns inverse problems with unknown topology, lengths of edges and potentials. Belishev [8] and Belishev and Vakulenko [9] used the spectral data, i.e. eigenvalues and the traces of the derivatives of eigenfunctions at the boundary vertices (that is equivalent to the knowledge of the TW-function).

The paper by Avdonin and Kurasov [3] contains the most complete results in this direction. It proves that a quantum tree is uniquely determined by the reduced TW-function associated to all except one boundary vertices. Moreover,

Key words and phrases. wave equation, boundary control method, Titchmarsh-Weyl function, leaf peeling method.

This research was supported in part by the Institute for Mathematics and its Applications with funds provided by the National Science Foundation and by the Ministry of Education and Science of Republic of Kazakhstan under the grant No. 4290/GF4.

Sergei Avdonin was supported by NSF grant DMS 1411564 .

Victor Mikhaylov was supported by RFBR 14-01-00535, RFBR 14-01-31388 and NIR SPbGU 11.38.263.2014. 
the reduced response operator (the dynamical Dirichlet-to-Neumann map) known on sufficiently large time interval also determines a tree uniquely. The expression "sufficiently large" means precisely the time interval of exact controllability of the tree.

The most significant result of $[\mathbf{3}]$ is developing a constructive and robust procedure for the recovery tree's parameters, which became known as the leaf peeling (LP) method. This method was extended to boundary inverse problems with nonstandard vertex conditions in $[\mathbf{4}]$, to the two-velocity wave equation in $[\mathbf{5}, \mathbf{6}]$ and to the parabolic type equations on trees in $[\mathbf{2}]$.

Our procedure is recursive - it allows recalculating efficiently the TW-function (and response operator) from the original tree to the smaller trees, 'removing' leaves step by step up to the rooted edge. Because of its recursive nature, this procedure contains only Volterra type equations, and hence may be a base for developing effective numerical algorithms. The fact that the proposed procedure is recursive is crucial for its numerical realization since the number of edges of graphs arising in applications is typically very big.

The development of effective numerical algorithms for solving inverse problems on quantum graphs is one of the goals of our research of quantum graphs. The first results in this direction for a star graph were obtained in [1]. The LP method combines both spectral and dynamical approaches to inverse problems. However, the main step of the LP algorithm - recalculating the inverse data for the 'peeled tree' - was described in detail only in a spectral version. In this paper we describe its dynamical version, very important from theoretical and numerical viewpoints.

Let $\Omega$ be a finite connected compact graph without cycles (a tree). The graph consists of edges $E=\left\{e_{1}, \ldots, e_{N}\right\}$ connected at the vertices $V=\left\{v_{1} \ldots, v_{N+1}\right\}$. Every edge $e_{j} \in E$ is identified with an interval $\left(a_{2 j-1}, a_{2 j}\right)$ of the real line. The edges are connected at the vertices $v_{j}$ which can be considered as equivalence classes of the edge end points $\left\{a_{j}\right\}$. The boundary $\Gamma=\left\{\gamma_{1}, \ldots, \gamma_{m}\right\}$ of $\Omega$ is a set of vertices having multiplicity one (the exterior nodes). Since the graph under consideration is a tree, for every $a, b \in \Omega, a \neq b$, there exists the unique path $\pi[a, b]$ connecting these points.

\section{Spectral and dynamical problems, inverse data.}

Let $\partial w\left(a_{j}\right)$ denotes the derivative of $w$ at the vertex $a_{j}$ taken along the corresponding edge in the direction outward the vertex. We associate the following spectral problem on the graph $\Omega$ with the potential $q \in L_{1}(\Omega)$ :

$$
\begin{array}{r}
-\frac{d^{2} w}{d x^{2}}+q w=\lambda w, \\
w \in C(\Omega), \\
\sum_{e_{j} \sim v} \partial_{j} w(v)=0 \quad \text { for } v \in V \backslash \Gamma, \\
w=0 \quad \text { on } \Gamma .
\end{array}
$$

In $(2.3) \partial_{j} w(v)$ denotes the derivative of $w$ at the vertex $v$ taken along the edge $e_{j}$ in the direction outwards the vertex. Also, $e_{j} \sim v$ means edge $e_{j}$ is incident to vertex $v$, and the sum is taken over all edges incident to $v$.

It is well-known fact that the problem (2.1)-(2.4) has a discrete spectrum of eigenvalues $\lambda_{1} \leqslant \lambda_{2} \leqslant \ldots, \lambda_{k} \rightarrow+\infty$, and corresponding eigenfunctions $\phi_{1}, \phi_{2}, \ldots$ 
can be chosen so that $\left\{\phi_{k}\right\}_{k=1}^{\infty}$ forms an orthonormal basis in $\mathcal{H}:=L_{2}(\Omega)$ :

$$
\left(\phi_{i}, \phi_{j}\right)_{\mathcal{H}}=\int_{\Omega} \phi_{i}(x) \phi_{j}(x) d x=\delta_{i j} .
$$

Set $\varkappa_{k}(\gamma)=\partial \phi_{k}(\gamma), \gamma \in \Gamma$. Let $\alpha_{k}$ be the $m$-dimensional column vector defined as $\alpha_{k}=\operatorname{col}\left(\frac{\varkappa_{k}(\gamma)}{\sqrt{\lambda_{k}}}\right)_{\gamma \in \Gamma}$.

DeFinition 1. The set of pairs

$$
\left\{\lambda_{k}, \alpha_{k}\right\}_{k=1}^{\infty}
$$

is called the Dirichlet spectral data of the graph $\Omega$.

The inverse spectral problem with the data given by (2.5) was considered in [8]. We will be dealing with the Titchmarsh-Weyl function which is defined as follows. We consider the differential equation on $\Omega$ for $\lambda \notin \mathbb{R}$ :

$$
-\phi^{\prime \prime}(x)+q(x) \phi(x)=\lambda \phi(x) .
$$

Let the function $\psi_{i}(x, \lambda)$ be the solution to $(2.6)$ with standard conditions (2.2), (2.3) at internal vertices and the following boundary conditions

$$
\psi_{i}\left(\gamma_{i}, \lambda\right)=1, \quad \psi_{i}\left(\gamma_{j}, \lambda\right)=0, \quad j \neq i .
$$

Then the entries of the Titchmarsh-Weyl matrix $\mathbf{M}(\lambda)$ are defined as

$$
M_{i j}(\lambda)=\partial \psi_{i}\left(\gamma_{j}\right) .
$$

Let $\mathrm{t} \psi$ be the solution to (2.1), (2.2) and (2.3) with the nonhomogeneous Dirichlet boundary conditions:

$$
\psi=\zeta \text { on } \Gamma,
$$

where $\zeta \in \mathbb{R}^{m}$. The Titchmarsh-Weyl $M$-matrix connects the values of $\psi$ on the boundary and the values of its derivative on the boundary:

$$
\partial \psi=\mathbf{M}(\lambda) \zeta \quad \text { on } \Gamma .
$$

Along with the spectral we consider the dynamical problem:

$$
\begin{array}{r}
u_{t t}-u_{x x}+q u=0 \quad \text { in } \Omega \backslash V \times[0, T], \\
\left.u\right|_{t=0}=\left.u_{t}\right|_{t=0}=0, \\
u(\cdot, t) \quad \text { satisfies (2.2) and (2.3) for all } t \in[0, T], \\
u=f \quad \text { on } \Gamma \times[0, T] .
\end{array}
$$

Here $T>0, f=f(\gamma, t), \gamma \in \Gamma$, is the Dirichlet boundary control which belongs to $\mathcal{F}_{\Gamma}^{T}:=L_{2}\left([0, T] ; \mathbb{R}^{m}\right)$. Let $u^{f}$ be the solution to the problem $(2.9)-(2.12)$ with the boundary control $f$. We introduce the dynamical response operator (the dynamical Dirichlet-to-Neumann map) by the rule

$$
\left(R^{T}\{f\}\right)(t)=\left.\partial u^{f}(\cdot, t)\right|_{\Gamma}, \quad t \in[0, T] .
$$

The response operator has a form of convolution:

$$
\left(R^{T}\{f\}\right)(t)=(\mathbf{R} * f)(t), t \in[0, T] .
$$

Here the matrix-valued response function $\mathbf{R}(t)$ is defined by the following procedure. Let the function $u_{i}(x, t)$ be the solution to $(2.9)-(2.11)$ and the boundary conditions

$$
u_{i}\left(\gamma_{i}, t\right)=\delta(t), \quad u_{i}\left(\gamma_{j}, t\right)=0, \quad j=1, \ldots m, j \neq i .
$$


The entries of response matrix $\mathbf{R}$ are defined by

$$
R_{i j}(t)=\partial u_{i}\left(\gamma_{j}, t\right)
$$

Connection between the spectral and dynamical data are known and were used for studying inverse spectral and dynamical problems, see for example $[\mathbf{3}, \mathbf{7}]$. Let $f \in \mathcal{F}_{\Gamma}^{T} \cap\left(C_{0}^{\infty}(0,+\infty)\right)^{m}$ and

$$
\widehat{f}(k):=\int_{0}^{\infty} f(t) e^{i k t} d t
$$

be its Fourier transform. The equations (2.9), and (2.1) are connected by the Fourier transformation: going formally in (2.9) over to the Fourier transform, we obtain (2.1) with $\lambda=k^{2}$. It is not difficult to check (see, e.g. $[\mathbf{3}, \mathbf{7}]$ ) that the response matrix-function and Titchmarsh-Weyl matrix are connected by the same transform:

$$
\mathbf{M}\left(k^{2}\right)=\int_{0}^{\infty} \mathbf{R}(t) e^{i k t} d t
$$

where this equality is understood in a weak sense.

\section{Inverse spectral problem. Recovering the Titchmarsh-Weyl function for the peeled tree.}

Any boundary vertex of the tree can be taken as a root; therefore without loss of generality, we can assume that the boundary vertex $\gamma_{m}$ is a root of the tree. We put $\Gamma_{m}=\Gamma \backslash\left\{\gamma_{m}\right\}$ and consider the spectral problem (2.1)-(2.4) on $\Omega$. The reduced TW matrix $\mathbf{M}(\lambda)=\left\{M_{i j}(\lambda)\right\}_{i, j=1}^{m-1}$ associated with boundary points from $\Gamma_{m}$ is constructed as in Section 1 and serve as data for our inverse problem.

Using the procedure described in $[\mathbf{3}]$ we can recover the potential on all the boundary edges (it suffices to know only the diagonal elements of the $M$-matrix to do it). Moreover, using the non-diagonal elements we can identify the sets of boundary edges incident to the same internal vertex. We call these sets pre-sheaves. More precisely, we introduce the following

DEFINITION 2. We consider a subgraph of $\Omega$ which is a star graph consisting of all edges incident to an internal vertex $v$. This star graph is called a pre-sheaf if it contains at least one boundary edge of $\Omega$. A pre-sheaf is called a sheaf if all but one its edges are the boundary edges of $\Omega$.

Following [6] one can extract a sheaf from all pre-sheaves found on the previous step and proceed with the leaf-peeling method procedure described below.

Let the found sheaf consist of the boundary vertices $\gamma_{1}, \ldots, \gamma_{m_{0}}$ from $\Gamma_{m}$, the corresponding boundary edges $e_{1}, \ldots, e_{m_{0}}$ and an internal edge $e_{m_{0}^{\prime}}$. We identify each edge $e_{i}, i=1, \ldots, m_{0}$, with the interval $\left[0, l_{i}\right]$ and the vertex $\gamma_{m_{0}^{\prime}}$, the internal vertex of the sheaf, - with the set of common endpoints $x=0$. At this point it is convenient to renumerate the edge $e_{m_{0}^{\prime}}$ as $e_{0}$ and the vertex $\gamma_{m_{0}^{\prime}}$ as $\gamma_{0}$. Applying the techniques from $[\mathbf{3}, \mathbf{6}]$ we recover the potential $q$ and lengths $l_{i}$ of edges $e_{i}$, $i=1, \ldots, m_{0}$.

We call $\widetilde{\mathbf{M}}(\lambda)$ the reduced $M$-matrix associated with the new graph $\widetilde{\Omega}=$ $\Omega \backslash \bigcup_{i=1}^{m_{0}}\left\{e_{i}, \gamma_{i}\right\}$ with boundary points $\Gamma \backslash \bigcup_{i=1}^{m_{0}} \gamma_{i}$.

First we recalculate entries $\widetilde{M}_{0 i}(\lambda), i=0, m_{0}+1, \ldots, m-1$. Let us fix $\gamma_{1}$, the boundary point of the star-subgraph. Let $u$ be the solution to the problem (2.6) 
with the boundary conditions

$$
u\left(\gamma_{1}\right)=1, \quad u\left(\gamma_{j}\right)=0, \quad j=2, \ldots, m
$$

We point out that on the boundary edge $e_{1}$ the function $u$ solves the Cauchy problem

$$
\left\{\begin{array}{l}
-u^{\prime \prime}(x)+q(x) u(x)=\lambda u(x), \quad x \in e_{1}, \\
u\left(l_{1}\right)=1, u^{\prime}\left(l_{1}\right)=M_{11}(\lambda)
\end{array}\right.
$$

On other boundary edges of the sheaf it solves the problems

$$
\left\{\begin{array}{l}
-u^{\prime \prime}(x)+q(x) u(x)=\lambda u(x) \\
u\left(l_{i}\right)=0, u^{\prime}\left(l_{i}\right)=M_{1 i}(\lambda)
\end{array} \quad, \quad x \in e_{i}, \quad i=2, \ldots, m_{0}\right.
$$

Since we know the potential on the edges $e_{1}, \ldots, e_{m_{0}}$, we can solve the Cauchy problems (3.1) and (3.2), and use the conditions (2.2), (2.3) at the internal vertex $v_{0}$ to recover $u(0, \lambda), u^{\prime}(0, \lambda)$ - the values of the solution and its derivative at the "new" boundary edge with the "new" boundary point $v_{0}$. Thus we obtain:

$$
\begin{array}{r}
\widetilde{M}_{00}(\lambda)=\frac{u^{\prime}(0, \lambda)}{u(0, \lambda)}, \\
\widetilde{M}_{0 i}(\lambda)=\frac{M_{1 i}(\lambda)}{u(0, \lambda)}, \quad i=m_{0}+1, \ldots m-1 .
\end{array}
$$

To find $\widetilde{M}_{i 0}(\lambda), i=m_{0}+1, \ldots, m-1$ we fix $\gamma_{i}, i \notin\left\{1, \ldots, m_{0}, m\right\}$ and consider the solution $u$ to $(2.6)$ with the boundary conditions

$$
u\left(\gamma_{i}\right)=1, \quad u\left(\gamma_{j}\right)=0, \quad j \neq i .
$$

The function $u$ solves the Cauchy problems on the edges $e_{1}, \ldots, e_{m_{0}}$ :

$$
\left\{\begin{array}{l}
-u^{\prime \prime}(x)+q(x) u(x)=\lambda u(x) \\
u\left(\gamma_{j}\right)=0, u^{\prime}\left(\gamma_{j}\right)=M_{i j}(\lambda)
\end{array} \quad, \quad x \in e_{j}, \quad j=1, \ldots, m_{0} .\right.
$$

Since we know the potential on the edges of the subgraph, we can solve the Cauchy problems (3.3) and use the conditions at the internal vertex $v_{0}$ to recover $u(0, \lambda)$, $u^{\prime}(0, \lambda)$ - the values of solution and its derivative at the "new" boundary edge with the "new" boundary point $v_{0}$. On the subgraph $\widetilde{\Omega}$ the function $u$ solves equation (2.6) with the boundary conditions

$$
u\left(\gamma_{i}\right)=1, u\left(\gamma_{0}\right)=u(0, \lambda), u\left(\gamma_{j}\right)=0, j=m_{0}+1, \ldots, m, \gamma_{j} \neq \gamma_{i}, \gamma_{j} \neq \gamma_{0} .
$$

Thus we obtain the equalities

$$
\begin{gathered}
\widetilde{M}_{i 0}(\lambda)=u_{x}(0, \lambda)-u(0, \lambda) \widetilde{M}_{00}(\lambda), \\
\widetilde{M}_{i j}(\lambda)=M_{i j}(\lambda)-u(0, \lambda) \widetilde{M}_{0 j}(\lambda) .
\end{gathered}
$$

To recover all elements of the reduced matrix we need to use this procedure for all $i, j=m_{0}+1, \ldots, m-1$.

Thus using the described procedure we can identify a sheaf and recalculate the truncated TW-matrix for the new 'peeled' tree, i.e. a tree without this sheaf. Repeating the procedure sufficient number of times, we recover the topology of a tree and a potential. 


\section{Inverse dynamical problem. Recovering the response operator for the peeled tree.}

We assume that the boundary vertex $\gamma_{m}$ is a root of the tree. We put $\Gamma_{m}=$ $\Gamma \backslash\left\{\gamma_{m}\right\}$ and consider the dynamical problem (2.9)-(2.12) on $\Omega$. The reduced response matrix function $\mathbf{R}(t)=\left\{R_{i j}(t)\right\}_{i, j=1}^{m-1}$ associated with boundary points from $\Gamma_{m}$ is constructed as in Section 1 and serves as inverse data.

We use the procedure described in $[\mathbf{3}, \mathbf{5}, \mathbf{6}]$ to recover the potential and lengths on all boundary edges and determine all sheaves.

Take the sheaf consisting of the boundary vertices $\gamma_{1}, \ldots, \gamma_{m_{0}}$ from $\Gamma_{m}$, the corresponding boundary edges $e_{1}, \ldots, e_{m_{0}}$ and an internal edge $e_{m_{0}^{\prime}}$. We identify each edge $e_{i}, i=1, \ldots, m_{0}$, with the interval $\left[0, l_{i}\right]$ and the vertex $\gamma_{m_{0}^{\prime}}$, the internal vertex of the sheaf, - with the set of common endpoints $x=0$. At this point it is convenient to renumerate the edge $e_{m_{0}^{\prime}}$ as $e_{0}$ and the vertex $\gamma_{m_{0}^{\prime}}$ as $\gamma_{0}$.

We call $\widetilde{\mathbf{R}}(t)$ the reduced response function associated with the new graph $\widetilde{\Omega}=\Omega \backslash \bigcup_{i=1}^{m_{0}}\left\{e_{i}, \gamma_{i}\right\}$ and with boundary points $\Gamma \backslash \bigcup_{i=1}^{m_{0}} \gamma_{i}$.

To recover the entries $\widetilde{R}_{0 i}(t), i=m_{0}+1, \ldots, m-1$, we consider the function $u^{\delta}$ to be the solution to the problem $(2.9),(2.10),(2.11)$ with the boundary conditions

$$
u^{\delta}\left(\gamma_{1}, t\right)=\delta(t), \quad u^{\delta}\left(\gamma_{j}, t\right)=0, \quad j=2, \ldots, m .
$$

Since we know the potential on the edges $e_{1}, \ldots, e_{m_{0}}$, we can evaluate $u^{\delta}$ on these edges by solving the wave equations on the corresponding edges with the known boundary data. Let us introduce the functions:

$$
\begin{array}{r}
F_{1}(t)=\delta(t), \quad F_{i}(t)=0, \quad i=2, \ldots, m_{0}, \quad t \in \mathbb{R}, \\
R_{1}(t)=R_{11}(t), t>0, \quad R_{i}(t)=\left\{\begin{array}{l}
0, \quad t<l_{1}+l_{i} \\
R_{1 i}(t), \quad t \geqslant l_{1}+l_{i},
\end{array} \quad i=2 \ldots, m_{0} .\right.
\end{array}
$$

Then the function $u^{\delta}$ solves the following Cauchy problems on $e_{1}, \ldots, e_{m_{0}}$ :

$$
\left\{\begin{array}{l}
u_{t t}^{\delta}-u_{x x}^{\delta}+q(x) u^{\delta}=0, x \in\left(0, l_{i}\right) \\
u^{\delta}\left(l_{i}, t\right)=F_{i}(t), \quad u_{x}^{\delta}\left(l_{i}, t\right)=R_{i}(t) \quad, \quad i=1 \ldots, m_{0} \\
u^{\delta}(x, 0)=0, x \in\left(0, l_{i}\right)
\end{array}\right.
$$

Using the compatibility conditions $(2.10),(2.11)$ at the vertex $v_{0}$ we can find the values of $u^{\delta}(0, t)$ and $u_{x}^{\delta}(0, t)$ for $t>0$ at the "new" boundary edge $e_{0}$. We introduce the notations

$$
a(t):=u^{\delta}(0, t), \quad A(t):=u_{x}^{\delta}(0, t),
$$

where $a(t)=A(t)=0$ for $t<l_{1}$.

Let us now consider $u^{f}$ to be the solution to the problem (2.9), (2.10), (2.11) with the boundary conditions

$$
u^{f}\left(\gamma_{1}, t\right)=f(t), \quad u^{f}\left(\gamma_{j}, t\right)=0, \quad j=2, \ldots, m .
$$

Due to Duhamel's principle, $u^{f}(\cdot, t)=\left(u^{\delta} * f\right)(\cdot, t)$, and at the "new" boundary vertex we have:

$$
u^{f}\left(\gamma_{0}, t\right)=a(t) * f(t) .
$$

By the definitions of the response matrices $\mathbf{R}, \widetilde{\mathbf{R}}$, the following equalities are valid:

$$
\int_{0}^{t} R_{1 i}(s) f(t-s) d s=\int_{0}^{t} \widetilde{R}_{0 i}(s)[a * f](t-s) d s, \quad i=m_{0}+1, \ldots, m-1 .
$$


It was proved in [2] that the response operator $R^{T}$, known for sufficiently large $T$, uniquely determines the spectral data and the Titchmarsh-Weyl matrix function. 'Sufficiently large' means precisely that $T$ is not less than the time of exact controllability: $T \geq 2$ dist $\left\{\gamma_{m}, \Gamma_{m}\right\}$. In $[\mathbf{2}]$ the inverse problem for the heat equation with the Neumann-to-Dirichlet data was studied, but the proof extends to our problem. Therefore, we assume below that all sums are finite, since we may consider the corresponding functions on finite time intervals.

We know (see, e.g. [3]) that $R_{1 i}(s), \widetilde{R}_{0 i}(s), i=m_{0}+1, \ldots, m-1$, and $a$ admit the following representations (we separate regular and singular parts):

$$
\begin{aligned}
& R_{1 i}(s)=r_{1 i}(s)+\sum_{n \geqslant 1} \alpha_{n} \delta^{\prime}\left(s-\beta_{n}\right),\left.\quad r_{1 i}\right|_{s \in\left(0, \beta_{1}\right)}=0 \\
& a(s)= \widetilde{a}(s)+\sum_{k \geqslant 1} \psi_{k} \delta\left(s-\varkappa_{k}\right),\left.\quad \widetilde{a}\right|_{s \in\left(0, \varkappa_{1}\right)}=0, \varkappa_{1}=l_{1}, \\
& \widetilde{R}_{0 i}(s)=\widetilde{r}_{0 i}(s)+\sum_{l \geqslant 1} \phi_{l} \delta^{\prime}\left(s-\zeta_{l}\right),\left.\quad \widetilde{r}_{0 i}\right|_{s \in\left(0, \zeta_{1}\right)}=0 .
\end{aligned}
$$

In the above representations the function $\widetilde{r}_{b i}(s)$, and numbers $\psi_{l}$ and $\zeta_{l}$ are unknown and sequences $\beta_{n}, \varkappa_{n}, \zeta_{n}$ are strictly increasing. Plugging these representations to (4.1), we obtain the following expression for the left hand side of (4.1):

$$
\int_{0}^{t} R_{1 i}(s) f(t-s) d s=\int_{0}^{t} r_{1 i}(s) f(t-s) d s-\sum_{n \geqslant 1} \alpha_{n} f^{\prime}\left(t-\beta_{n}\right) .
$$

For the right hand side of (4.1) we have:

$$
\begin{array}{r}
\int_{0}^{t} \widetilde{R}_{0 i}(s)[a * f](t-s) d s=\int_{0}^{t} \widetilde{r}_{0 i}(s) \int_{0}^{t-s} \widetilde{a}(\tau) f(t-s-\tau) d \tau+ \\
\sum_{k \geqslant 1} \psi_{k} \int_{0}^{t} \widetilde{r}_{0 i}(s) f\left(t-s-\varkappa_{k}\right) d s-\sum_{l \geqslant 1} \phi_{l} \int_{0}^{t-\zeta_{l}} \widetilde{a}^{\prime}(\tau) f\left(t-\zeta_{l}-\tau\right) d \tau- \\
\sum_{l \geqslant 1} \sum_{k \geqslant 1} \phi_{l} \psi_{k} f^{\prime}\left(t-\zeta_{l}-\varkappa_{k}\right) .
\end{array}
$$

Equating singular parts of the integral kernels of (4.2) and (4.3), we obtain:

$$
\sum_{n \geqslant 1} \alpha_{n} \delta^{\prime}\left(t-\beta_{n}\right)=\sum_{l \geqslant 1} \sum_{k \geqslant 1} \phi_{l} \psi_{k} \delta^{\prime}\left(t-\zeta_{l}-\varkappa_{k}\right) .
$$

The equation (4.4) allows one to recover the unknown coefficients $\zeta_{l}$ and $\phi_{l}$. Equating the first terms in (4.4), we necessarily have that

$$
\beta_{1}=\zeta_{1}+\varkappa_{1}, \quad \alpha_{1}=\psi_{1} \phi_{1}
$$

and so,

$$
\zeta_{1}=\beta_{1}-\varkappa_{1}, \quad \phi_{1}=\frac{\alpha_{1}}{\psi_{1}},
$$

The fact that the set $\left\{\alpha_{1}, \beta_{1}\right\},\left\{\psi_{1}, \varkappa_{1}\right\}$ determines $\left\{\phi_{1}, \zeta_{1}\right\}$ we represent in the following form:

$$
\left\{\alpha_{k}, \beta_{k}\right\}_{k=1}^{N_{1}},\left\{\psi_{k}, \varkappa_{k}\right\}_{k=1}^{N_{1}} \Longrightarrow\left\{\phi_{1}, \zeta_{1}\right\}, \quad m_{1}=1, \quad N_{1}=m_{1}
$$

Considering the second term in the left hand side in (4.4), we compare $\beta_{2}$ and $\zeta_{1}+\varkappa_{2}$. We get the options: 
1) In the case $\beta_{2} \neq \zeta_{1}+\varkappa_{2}$ we conclude that $\beta_{2}=\zeta_{2}+\varkappa_{1}$ and thus

$$
\zeta_{2}=\beta_{2}-\varkappa_{1}, \quad \phi_{2}=\frac{\alpha_{2}}{\psi_{1}}, \quad m_{2}=1 .
$$

2) In the case $\zeta_{1}+\varkappa_{2}=\beta_{2}$, but $\alpha_{2} \neq \phi_{1} \psi_{2}$ we have that $\zeta_{2}+\varkappa_{1}=\zeta_{1}+\varkappa_{2}=\beta_{2}$ and $\alpha_{2}=\phi_{1} \psi_{2}+\phi_{2} \psi_{1}$, so

$$
\zeta_{2}=\beta_{2}-\varkappa_{1}, \quad \phi_{2}=\frac{\alpha_{2}-\phi_{1} \psi_{2}}{\psi_{1}}, \quad m_{2}=1 .
$$

3) In the case $\zeta_{1}+\varkappa_{2}=\beta_{2}$ and $\alpha_{2}=\phi_{1} \psi_{2}$ we need to consider the third term in the left hand side of (4.4) and compare $\beta_{3}$ with $\zeta_{1}+\varkappa_{3}$, using the same procedure.

Repeating this procedure sufficient number of times (say, $m_{2}$ ), we recover $\left\{\phi_{2}, \zeta_{2}\right\}$. Suppose that to recover $\left\{\phi_{2}, \zeta_{2}\right\}$ we used the coefficients $\left\{\alpha_{k}, \beta_{k}\right\}_{k=1}^{N_{2}},\left\{\psi_{k}, \varkappa_{k}\right\}_{k=1}^{N_{2}}$; we express this fact in the form

$$
\left\{\alpha_{k}, \beta_{k}\right\}_{k=1}^{N_{2}},\left\{\psi_{k}, \varkappa_{k}\right\}_{k=1}^{N_{2}} \Longrightarrow\left\{\phi_{k}, \zeta_{k}\right\}_{k=1}^{2}, \quad N_{2}=N_{1}+m_{2}
$$

Assume that we have already recovered $p$ pairs:

$$
\left\{\alpha_{k}, \beta_{k}\right\}_{k=1}^{N_{p}},\left\{\psi_{k}, \varkappa_{k}\right\}_{k=1}^{N_{p}} \Longrightarrow\left\{\phi_{k}, \zeta_{k}\right\}_{k=1}^{p} .
$$

To recover $\left\{\phi_{p+1}, \zeta_{p+1}\right\}$ we need to apply the procedure described above: consider $N_{p}+1$-th term in the left hand side of (4.4) and compare $\beta_{N_{p}+1}$ with $\zeta_{p}+\varkappa_{N_{p}+1}$ to get the options:

1) If $\zeta_{p}+\varkappa_{N_{p}+1} \neq \beta_{N_{p}+1}$, then $\beta_{N_{p}+1}=\zeta_{p+1}+\varkappa_{N_{p}}$, thus

$$
\zeta_{p+1}=\beta_{N_{p}+1}-\varkappa_{N_{p}}, \quad \phi_{p+1}=\frac{\alpha_{N_{p}+1}}{\psi_{N_{p}}}, \quad m_{p+1}=1 .
$$

2) In the case $\beta_{N_{p}+1}=\zeta_{p}+\varkappa_{N_{p}+1}$, but $\alpha_{N_{p}+1} \neq \phi_{p} \psi_{N_{p}+1}$, we conclude that $\beta_{N_{p}+1}=\zeta_{p+1}+\varkappa_{N_{p}}=\zeta_{p}+\varkappa_{N_{p}+1}$ and $\alpha_{N_{p}+1}=\phi_{p} \psi_{N_{p}+1}+\phi_{p+1} \psi_{N_{p}}$. Thus we get

$$
\zeta_{p+1}=\beta_{N_{p}+1}-\varkappa_{N_{p}}, \quad \phi_{p+1}=\frac{\alpha_{N_{p}+1}-\phi_{p} \psi_{N_{p}+1}}{\psi_{N_{p}}}, \quad m_{p+1}=1 .
$$

3) If $\zeta_{p}+\varkappa_{N_{p}+1}=\beta_{N_{p}+1}$ and $\alpha_{N_{p}+1}=\phi_{p} \psi_{N_{p}+1}$ we need to compare $\zeta_{p}+$ $\varkappa_{N_{p}+2}$ with $\beta_{N_{p}+2}$ using the same procedure.

Repeating this procedure sufficient number of times (say, $m_{p+1}$ ), we recover $\left\{\phi_{p+1}, \zeta_{p+1}\right\}$. We write this in the form

$$
\left\{\alpha_{k}, \beta_{k}\right\}_{k=1}^{N_{p+1}},\left\{\psi_{k}, \varkappa_{k}\right\}_{k=1}^{N_{p+1}} \Longrightarrow\left\{\phi_{k}, \zeta_{k}\right\}_{k=1}^{p+1}, \quad N_{p+1}=N_{p}+m_{p+1} .
$$

The more quadruplets $\{\beta, \varkappa, \alpha, \phi\}$ we know, the more pairs $\{\phi, \zeta\}$ we can evaluate. Certainly the number of quadruplets at our disposal depends on the time interval at which we know the inverse data. In the case when we know the response function on $(0,+\infty)$, using the procedure described above, we can recover $\left\{\phi_{k}, \zeta_{k}\right\}$ for arbitrary $k$. 
Taking in (4.2), (4.3) $f(t)=\Theta(t)$ the Heaviside function, and equating the regular parts, we arrive at

$$
\begin{array}{r}
\int_{0}^{t} r_{1 i}(s) d s=\int_{l_{1}}^{t} \widetilde{r}_{0 i}(s) \int_{0}^{t-s} \widetilde{a}(\tau) d \tau d s \\
+\sum_{k \geqslant 1} \psi_{k} \int_{0}^{t-\varkappa_{k}} \widetilde{r}_{0 i}(s) d s-\sum_{l \geqslant 1} \phi_{l} \int_{0}^{t-\zeta_{l}} \widetilde{a}^{\prime}(\tau) d \tau
\end{array}
$$

Everywhere below we assume that all functions are extended to the interval $(-\infty, 0)$ by zero. Differentiating the last equality we get

$$
r_{1 i}(t)=\int_{0}^{t} \widetilde{r}_{0 i}(s) \widetilde{a}(t-s) d s+\sum_{k \geqslant 1} \psi_{k} \widetilde{r}_{0 i}\left(t-\varkappa_{k}\right)-\sum_{l \geqslant 1} \phi_{l} \widetilde{a}^{\prime}\left(t-\zeta_{l}\right),
$$

We set $s:=t-\varkappa_{1}$ and rewrite (4.5) as

$$
\begin{array}{r}
r_{1 i}\left(s+\varkappa_{1}\right)=\int_{0}^{s+\varkappa_{1}} \widetilde{r}_{0 i}(\tau) \widetilde{a}\left(s+\varkappa_{1}-\tau\right) d \tau+\psi_{1} \widetilde{r}_{0 i}(s)+ \\
\sum_{k \geqslant 2} \psi_{k} \widetilde{r}_{0 i}\left(s+\varkappa_{1}-\varkappa_{k}\right)-\sum_{l} \phi_{l} \widetilde{a}^{\prime}\left(s+\varkappa_{1}-\zeta_{l}\right) .
\end{array}
$$

Let us introduce the number

$$
\alpha:=\min _{i \geqslant 1}\left(\varkappa_{i+1}-\varkappa_{i}\right) .
$$

Notice that $\alpha$ is a positive number, since we are dealing with the problem on a finite time interval. steps:

The integral equation (4.6) for the unknown function $\widetilde{r}_{m_{0}^{\prime} i}$ can be solved by

1) On the interval $\left(0, \zeta_{1}\right)$ we have: $\widetilde{r}_{0 i}(s)=0$.

2) On the interval $\left(\zeta_{1}, \zeta_{1}+\alpha\right)$ equation (4.6) has the form

$$
\begin{array}{r}
\int_{0}^{s+\varkappa_{1}} \widetilde{r}_{0 i}(\tau) \widetilde{a}\left(s+\varkappa_{1}-\tau\right) d \tau+\psi_{1} \widetilde{r}_{0 i}(s)=B(s), \\
B(s)=r_{1 i}\left(s+\varkappa_{1}\right)+\sum_{l} \phi_{l} \widetilde{a}^{\prime}\left(s+\varkappa_{1}-\zeta_{l}\right),
\end{array}
$$

where $B(s)$ is known for $s \in\left(\zeta_{1}, \zeta_{1}+\alpha\right)$.

3) On the interval $\left(\zeta_{1}+\alpha, \zeta_{1}+2 \alpha\right)$ equation (4.6) has form (4.7) where

$$
B(s)=-\psi_{2} \widetilde{r}_{0 i}\left(s+\varkappa_{1}-\varkappa_{2}\right)+r_{1 i}\left(s+\varkappa_{1}\right)+\sum_{l \geqslant 1} \phi_{l} \widetilde{a}^{\prime}\left(s+\varkappa_{1}-\zeta_{l}\right)
$$

is known function on $\left(\zeta_{1}+\alpha, \zeta_{1}+2 \alpha\right)$.

4) On the interval $\left(\zeta_{1}+n \alpha, \zeta_{1}+(n+1) \alpha\right)$ equation (4.6) has form (4.7) where

$$
B(s)=-\sum_{k=2}^{n+1} \psi_{k} \widetilde{r}_{0 i}\left(s+\varkappa_{1}-\varkappa_{k}\right)+r_{1 i}\left(s+\varkappa_{1}\right)+\sum_{l \geqslant 1} \phi_{l} \widetilde{a}^{\prime}\left(s+\varkappa_{1}-\zeta_{l}\right)
$$

is known function on $\left(\zeta_{1}+n \alpha, \zeta_{1}+(n+1) \alpha\right)$.

To recover $\widetilde{R}_{00}(s)$ one need to use the following equation:

$$
\int_{0}^{t} \widetilde{R}_{00}(s)[a * f](t-s) d s=u_{x}^{f}\left(v_{m_{0}^{\prime}}, t\right)=[A * f](t) .
$$


One need to repeat the procedure described above: write down the expansions for the $R_{00}(t), A(t), a(t)$ with singular and regular parts separated, determine the singular part and afterward, determine the regular part from corresponding integral equation.

To recover $\widetilde{R}_{i 0}(t)$, with $i$ fixed, $i=m_{0}+1, \ldots, m-1$ we consider $u^{f}$, the solution to the boundary value problem (2.9) with standard conditions at internal vertices $(2.10),(2.11)$ and the following boundary conditions:

$$
u^{f}\left(\gamma_{i}, t\right)=f(t), \quad u^{f}\left(\gamma_{j}, t\right)=0, \quad j=1, \ldots, m, j \neq i .
$$

Using the fact that we know the potential on the edges $e_{1}, \ldots, e_{m_{0}}$, we can recover the solution to the problem above by solving the Cauchy problem for the wave equations on the corresponding edges with known boundary data. Indeed, let us introduce the functions:

$$
\begin{array}{r}
F_{k}(t)=0, \quad k=1, \ldots, m_{0}, \quad t \geqslant 0, \\
R_{k}(t)=\left[R_{i k} * f\right](t), \quad t \geqslant 0 .
\end{array}
$$

The function $u^{f}$ solves the following Cauchy problems on $e_{1}, \ldots, e_{m_{0}}$ :

$$
\left\{\begin{array}{l}
u_{t t}^{f}-u_{x x}^{f}+q(x) u^{f}=0, \quad x \in\left(0, l_{k}\right), \\
u^{f}\left(\gamma_{i}, t\right)=F_{k}(t), \quad u_{x}^{f}\left(\gamma_{k}, t\right)=R_{k}(t), \quad k=1, \ldots, m_{0} . \\
u(x, 0)=0, x \in\left(0, l_{k}\right)
\end{array}\right.
$$

Using the compatibility conditions $(2.10),(2.11)$ at the vertex $v_{0}$ we find the values of $u^{f}(0, t)$ and $u_{x}^{f}(0, t)$ for $t>0$ at the new boundary vertex:

$$
a(t):=u^{f}(0, t), \quad A(t):=u_{x}^{f}(0, t) .
$$

Then using the definition of the response matrix (cf. (3.4)), we obtain the equalities

$$
\int_{0}^{t} \widetilde{R}_{i 0}(s) f(t-s) d s=A(t)-\left[R_{00} * a\right](t) .
$$

These equations for $R_{i 0}$ can be analyzed using the procedure described above: we represent $\widetilde{R}_{i 0}(t), A(t)$ and $a(t)$ as sums of regular and singular parts, determine the singular parts of $R_{i 0}$ and then determine the regular parts from corresponding integral equations by steps.

Concluding this section, we state that using the described procedure one can identify a sheaf and recalculate the truncated response function for the new 'peeled' tree, i.e. a tree without this sheaf. Repeating the procedure sufficient number of times, we recover the topology of the tree, potential and the lengths of the edges.

\section{References}

[1] S. Avdonin, B. Belinskiy and M. Matthews, Dynamical inverse problems on a metric tree, Inverse Problems 27(7), 1-21 (2011).

[2] S. Avdonin, B. Bell, Determining a distributed conductance parameter for a neuronal cable model defined on a tree graph, Journal of Inverse Problems and Imaging, (2015), 9, no. 3, 645-659.

[3] S. A. Avdonin and P. B. Kurasov, Inverse problems for quantum trees, Inverse Problems and Imaging. 2 (2008), no. 1, 1-21.

[4] Avdonin, Kurasov and Nowaczyk, On the reconstruction of boundary conditions for star graphs, Inverse Problems and Imaging, 4 (2010), no. 4, 579-598.

[5] S. Avdonin, G. Leugering and V. Mikhaylov, On an inverse problem for tree-like networks of elastic strings, Zeit. Angew. Math. Mech. 90, 136-150 (2010). 
[6] Avdonin S. A., A. Choque Rivero, G. Leugering, Mikhaylov V. S. . On the inverse problem of the two-velocity tree-like graph // Journal of Applied Mathematics and Mechanics. 2015. . DOI: 10.1002/zamm.201400126,

[7] S. A. Avdonin, V. S. Mikhaylov and A. V. Rybkin, The boundary control approach to the Titchmarsh-Weyl m-function, Comm. Math. Phys. 275 (2007), no. 3, 791-803.

[8] M.I. Belishev, Boundary spectral inverse problem on a class of graphs (trees) by the BC method, Inverse Problems 20 (2004), 647-672.

[9] M.I. Belishev, A.F. Vakulenko, Inverse problems on graphs: recovering the tree of strings by the BC-method, J. Inv. Ill-Posed Problems 14 (2006), 29-46.

[10] B. M. Brown and R. Weikard, A Borg-Levinson theorem for trees, Proc. R. Soc. Lond. Ser. A Math. Phys. Eng. Sci., 461 (2005), no. 2062, 3231-3243.

[11] G. Freiling and V. Yurko, Inverse problems for Strum-Liouville operators on noncompact trees, Results Math., 50 (2007), no. 3-4, 195-212.

[12] V. Yurko, Inverse Sturm-Liouville operator on graphs, Inverse Problems 21 (2005), 10751086.

Department of Mathematics and Statistics, University of Alaska Fairbanks, FairBANKS, AK 99775-6660, USA

E-mail address: s.avdonin@alaska.edu

St.Petersburg Department of V.A.Steklov Institute of Mathematics of the Russian Academy of Sciences, 7 Fontanka, St.Petersburg 191023, Russia; and St.-Petersburg State University, Faculty of Physics, University Embankment 7-9, St-Petersburg 199034, Russia

E-mail address: v.mikhaylov@spbu.ru

L.N. Gumilyov Eurasian National University, 2, Satpayev Str., Astana, 010008, KAZAKHSTAN

E-mail address: nurtazina.k@gmail.com 